\title{
Oxygen Vacancy Ordering: a Degree of Freedom that can Control the Structural, Electronic and Magnetic Properties of Transition-Metal Oxide Films.
}

\author{
M. Varela ${ }^{1,2}$, J. Salafranca ${ }^{2,1}$, N. Biskup ${ }^{2}$, J. Gazquez $^{3}$, M. P. Oxley ${ }^{4,1}$, V. Mehta ${ }^{5}$, Y. Suzuki ${ }^{5,6,7}$, S. \\ Bose $^{8}$, M. Sharma ${ }^{8}$, C. Leighton ${ }^{8}$, W.Luo ${ }^{4,1}$, S. T. Pantelides ${ }^{4,9,1}$, S. J. Pennycook ${ }^{10}$. \\ 1. Materials Science \& Technology Div., Oak Ridge National Laboratory, Oak Ridge TN 37831, U.S.A. \\ 2. Dept. de Física Aplicada III \& Instituto Pluridisciplinar, Univ. Complutense, Madrid, 28040 Spain. \\ 3. Instituto de Ciencia de Materiales de Barcelona, ICMAB-CSIC, Bellaterra, 08193 Spain. \\ 4. Dept. of Physics \& Astronomy, Vanderbilt University. Nashville, TN 37235, U.S.A. \\ 5. Dept. of Material Science and Engineering, University of California, Berkeley, CA 94720, U.S.A. \\ 6. Materials Sciences Division, Lawrence Berkeley National Laboratory, Berkeley, CA 94720, U.S.A. \\ 7. Dept. of Applied Physics and Geballe Laboratory for Advanced Materials, Stanford \\ University,Stanford, CA 94305, U.S.A. \\ 8. Dept. of Chemical Eng. and Materials Science, Univ. of Minnesota, Minneapolis, MN 55455, U.S.A \\ 9. Dept. of Electrical Eng. and Computer Science, Vanderbilt University, Nashville, TN 37235, U.S.A. \\ 10. Dept. of Materials Science \& Engineering, University of Tennessee, Knoxville TN 37996, U.S.A.
}

Oxygen vacancies are known to affect complex oxides, but current measurement techniques often are limited in that they provide "bulk views" of an entire sample, averaged over macroscopic length scales. Aberration corrected scanning transmission electron microscopy (STEM) and electron energy-loss spectroscopy (EELS) have demonstrated their ability to probe structural, chemical, and electronic properties with atomic-scale resolution in real space, and they have also provided magnetic information at the sub-nm scale in exquisite detail [1]. Here we combine atomically-resolved STEM-EELS with density-functional calculations to demonstrate that, in epitaxial cobaltite thin films, the combination of epitaxial strain along with ordered oxygen vacancies dominates the film's structure and electronic properties, promoting a long range magnetic ordering that is not present in the bulk.

Epitaxial lanthanum cobaltite $\left(\mathrm{LaCoO}_{3}\right.$ or $\left.\mathrm{LCO}\right)$ films under tensile strain, e.g., grown on SrTiO3, have attracted considerable interest as a test bed to understand the origins of unexpected physical phenomena. While the bulk material is non-magnetic, the films are ferromagnetic (FM) at low temperatures [2, 3]. Since the initial reports, the origin of the observed FM ordering has been debated extensively on the basis of theoretical calculations and complementary experimental data [4]. Competing interactions of comparable magnitude permit Co atoms to achieve low-spin (LS), intermediate spin (IS) or high-spin (HS) states [5, 6]. Different types of Co spin states and ordering, induced by homogeneous or inhomogeneous strain distributions, have been proposed. In these cases, the films have been assumed stoichiometric and no $\mathrm{O}$ deficiency has been considered. Here, we demonstrate that oxygen vacancy ordering in epitaxial LCO thin films can give rise to long range magnetic ordering accompanied by insulating behavior and a superlattice in the spin state as depicted in Figure 1 [7]. We show that epitaxial strain is relaxed through local lattice expansion at ordered oxygen-deficient atomic planes. The vacancies lead to excess electrons in the Co d-states, which order ferromagnetically. Finally, the expected metallic behavior caused by vacancy superstructure "doping" is disrupted by the appearance of Peierls-like minigaps that, on strain relaxation, lead to a radical change in the electronic structure, resulting in insulating behavior [7].

Such spin state superlattices not present in the bulk can also be stabilized by epitaxial strain in metallic 
compounds, such as doped $\mathrm{La}_{0.5} \mathrm{Sr}_{0.5} \mathrm{CoO}_{3-\mathrm{x}}$ (LSCO). The analysis of the near edge fine structure can provide a mapping of $\mathrm{Co}$ atom spin state with atomic resolution. In highly reduced nanopockets of $\mathrm{O}$ deficient LSCO thin films $[8,9]$, unexpected spin state superlattices associated with $\mathrm{O}$ vacancy ordering are observed, as shown in Figure 2. Such ordering phenomena are not observed in the bulk and, again, cannot be studied by diffraction techniques that provide spatial averages. These and other examples will be discussed in this talk in order to prove that oxygen vacancy ordering complements strain as a major controllable degree of freedom that can be used to engineer novel behavior in complex-oxide films.

[1] J. Salafranca et al., Nanoletters 12 (2012), 2499.

[2] D. Fuchs et al., Physical Review B 75 (2007), 144402.

[3] V. V. Mehta et al., J. Appl. Phys. 105 (2009), 07E503.

[4] J. M. Rondinelli, N. A. Spaldin, Physical Review B 79 (2009), 054409.

[5] J. B. Goodenough, Journal of Physics and Chemistry of Solids 6, (1958) 287.

[6] M. A. Senaris-Rodriguez, J. B. Goodenough, Journal of Solid State Chemistry 116 (1995), 224.

[7] N. Biskup et al., Physical Review Letters (2014), in press.

[8] J. Gazquez et al., Nanoletters 11 (2011), 973.

[9] J. Gazquez et al., Applied Physics Letters Materials 1 (2013), 012105

[10] The authors thank Masashi Watanabe for the Digital Micrograph PCA plug-in. Research at ORNL was supported by the U.S. Department of Energy (DOE), Basic Energy Sciences (BES), Materials Sciences and Engineering Division, and through a user project supported by ORNL's Center for Nanophase Materials CNMS) User Program, which is also sponsored by DOE-BES. Research at UCM was supported by the ERC starting Investigator Award, grant \#239739 STEMOX and the Juan de la Cierva program. Research at UC Berkeley/LBNL and Stanford was supported by the Director, Office of Science, Office of Basic Energy Sciences, Division of Materials Sciences and Engineering under Contract Numbers DE-AC02-05CH11231 and DE-SC0008505 respectively. Research at Vanderbilt was supported in part by the U.S DOE grant DE-FG02-09ER46554 and the McMinn Endowment. Computations were supported by the National Center for Supercomputing Applications (U.S. Department of Energy, contract no. DE-AC02-05CH11231). JG acknowledges support from the Spanish MEC 2007-0086. Work at UMN supported by NSF (DMR-0804432) and DOE (DE-FG02-06ER46275, specifically scattering characterization).

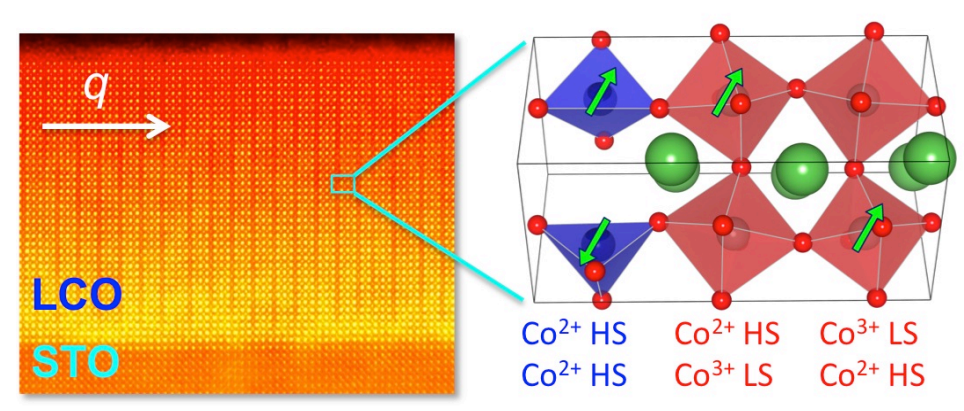

Figure 1. Z-contrast image of a LCO film grown on a STO substrate. The sketch on the right shows the structure and the magnetic order proposed by theory. $\mathrm{O}$ atoms are red, Co atoms are shown in blue, and $\mathrm{La}$ atoms are green. Adapted from reference 7 .

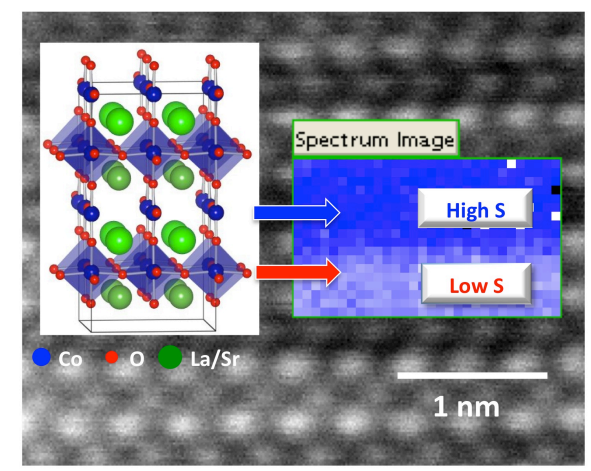

Figure 2. Z-contrast image of a LSCO thin film. The left inset is a schematic of the crystal structure; the right represents the $\mathrm{O}$ $K$ edge pre-peak intensity map, which correlates with the spin state. Adapted from reference [8]. 